\title{
Survey reveals health impact of downturn
}

$\mathrm{C}$ anadians are putting less effort and expense into maintaining their health as a result of the economic downtown, with lowincome householders cutting back on health-related activities and expenses more than their wealthier counterparts, according to a new survey by the Canadian Medical Association.

For all Canadians, concerns about health come a close second to worry about financial security. Some $57 \%$ of those surveyed report being somewhat or extremely worried about their financial security, while 52\% are similarly worried about their health, according to the survey released Aug. 17 at the CMA's annual meeting.

Meanwhile, the proportion of those giving health care services an "A" ranking is $26 \%$, up from $18 \%$ in a 2005 CMA survey, with those most likely to award the A being those aged 55 or older and living in urban areas of Ontario, Alberta and British Columbia.

Some $67 \%$ of those surveyed ranked health care services with grades of A or B, up from $63 \%$ in 2005 .

Though satisfaction with health care services may be slightly higher, the economic downturn is taking its toll on Canadian households.

Those in households earning less than $\$ 30000$ a year are more likely than those earning $\$ 90000$ or more to feel stressed and/or overwhelmed (48\% compared to $33 \%$ ); delay or stop buying prescription drugs (22\% compared to $7 \%$ ); delay or cancel a dentist appointment (34\% compared to $15 \%$ ), and sleep less than their normal amount (33\% compared to $16 \%$ ).

Those surveyed rated the top three factors that influence people's health status as tobacco use, stress and diet, while income level and education appeared much lower in the ranking. Epidemiological research generally reveals a much stronger association of income and education with health status, compared to the association between health and individual lifestyle

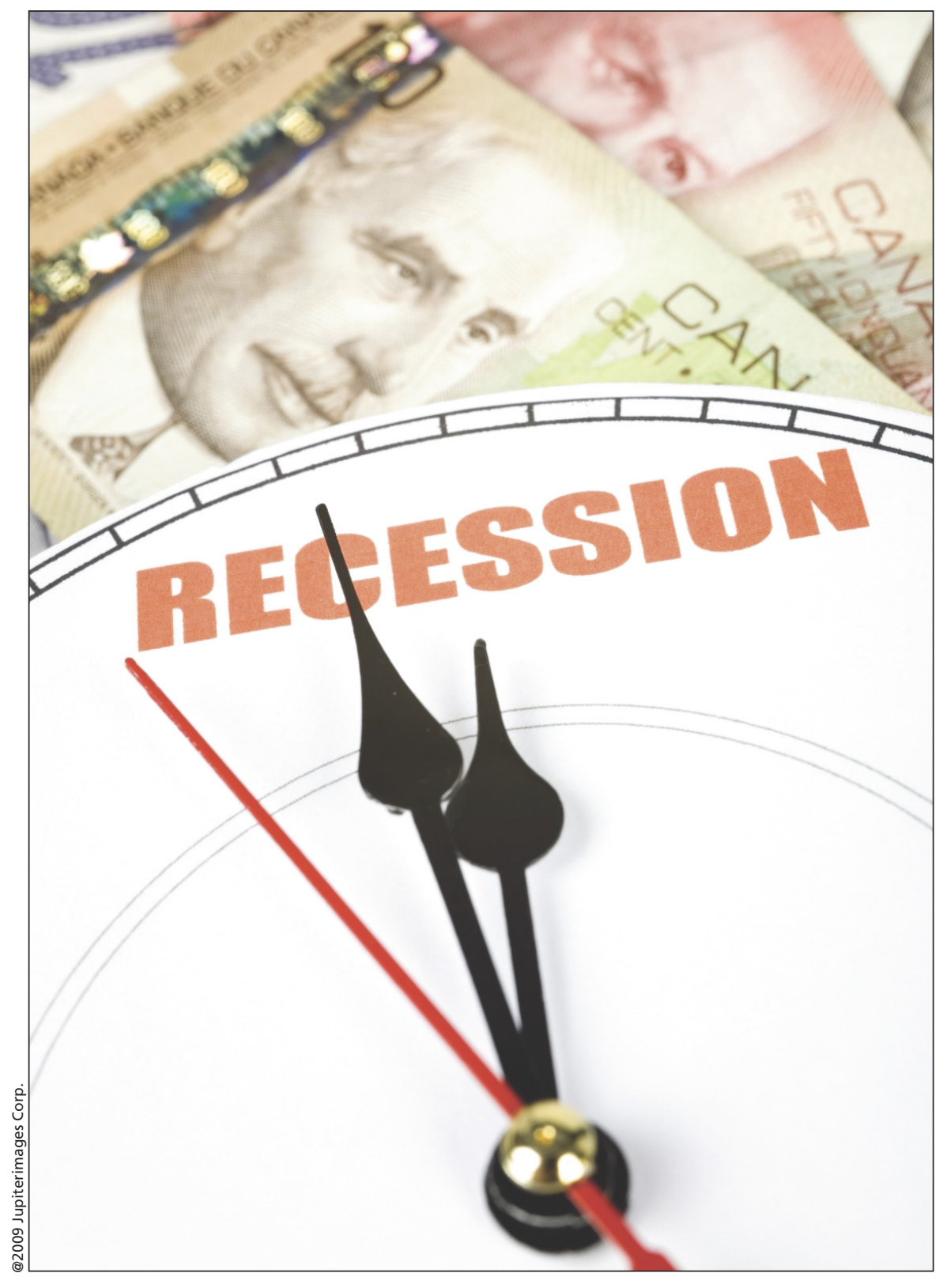

The economic downturn took its toll on Canadian households.

factors (see for example, A Healthy, Productive Canada: A Determinant of Health Approach, the June 2009 final report of the Senate Committee on Population Health co-chaired by Dr. Wilbert Keon).

Asked to estimate the extent to which a number of factors were important to health, survey participants stated that tobacco use had the greatest impact $(60 \%)$, followed by stress $(51 \%)$, diet and nutrition (49\%), income (27\%) and education level (18\%).

However, those with household incomes of less than $\$ 30000$ were more likely than those with household incomes greater than $\$ 30000$ to rate income as having an impact on health 
(36\%) compared to those with incomes over $\$ 90000(22 \%)$. They were also more likely to feel stressed or overwhelmed by financial concerns $(51 \%$ compared to $32 \%$ ), spend less on food (44\% compared to $23 \%$ ), and skip meals (28\% compared to $8 \%$ ).

The survey also found that Canadians with less than high school education were less likely to engage in "healthy living habits" such as eating breakfast $(60 \%$ compared to $83 \%)$, eating five servings of fruits and vegetables a day ( $32 \%$ compared to $56 \%)$, engaging in outdoor activities (37\% compared to $45 \%$ ), and sleeping six to eight hours a night $(65 \%$ compared to $82 \%$ ).
When asked to grade various aspects of the Canadian health care system, respondents issued marks fairly consistent with those assigned in previous CMA surveys. Improvements were reported in access to emergency ward services, access to healthcare on weekends and evenings, access to walk-in clinics and access to home health care service.

However, the percentage giving top marks for access to a family doctor declined from $35 \%$ in 2003 to $29 \%$.

Many respondents reported having to wait "longer than they thought was reasonable" for access to medical specialists, advanced diagnostic procedures and family physicians.
Ipsos Reid surveyed 3223 Canadian adults online between June 25 and July 11 and asked questions about their health status and social determinants of health. This sample size is associated with a $\pm 1.73 \%$ margin of error. The company surveyed 1002 Canadians by telephone between June 7 and June 9 and asked them to rate aspects of the health care system. This sample size is associated with a \pm 3.2 margin of error. The complete survey is available at: www.cma.ca/multimedia/CMA/Content _Images/Inside_cma/Media_Release/20 09/report_card/Report-Card_en.pdf). - Ann Silversides, CMAJ

DOI:10.1503/cmaj.109-3020 